\title{
Self-expandable metallic stent placement above the papilla without endoscopic sphincterotomy in patients with distal malignant biliary obstruction
}

다 (i) $(-)$

\author{
Authors \\ Institutions \\ 1 Department of Hepatobiliary and Pancreatic Oncology, \\ Osaka International Cancer Institute, Osaka, Japan \\ 2 Department of Cancer Survey and Gastrointestinal \\ Oncology, Osaka International Cancer Institute, Osaka, \\ Japan \\ 3 Cancer Control Center, Osaka International Cancer \\ Institute, Osaka, Japan
}

Ryoji Takada ${ }^{1}$, Kenji Ikezawa ${ }^{1}$, Ryosuke Kiyota ${ }^{1}$, Toshihiro Imai ${ }^{1}$, Yutaro Abe ${ }^{1}$, Nobuyasu Fukutake ${ }^{1}$, Reiko Ashida $^{2}$, Takatoshi Nawa', Takahiro Tabuchi ${ }^{3}$, Kazuhiro Katayama ${ }^{1,2}$, Kazuyoshi Ohkawa'

submitted 25.9.2019

accepted after revision 19.2.2020

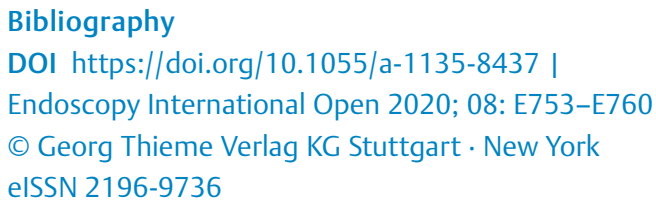

Corresponding author

Kenji Ikezawa, MD, PhD, Department of Hepatobiliary and Pancreatic Oncology, Osaka International Cancer Institute, 3-1-69 Otemae, Chuo-ku, Osaka 541-8567, Japan

Fax: +81-6-6945-1834

ikezawa-ke@mc.pref.osaka.jp

Supplementary material

Online content viewable at:

https://doi.org/10.1055/a-1135-8437

\section{ABSTRACT}

Background and study aims The best method for endoscopic placement of self-expandable metallic stents (SEMS) for distal malignant biliary obstruction (MBO) has not yet been determined. The aim of this study was to evaluate how SEMS placement above the papilla and without endoscopic sphincterotomy (EST) impacts the time to recurrent biliary obstruction (RBO) in patients with distal MBO.

Patients and methods We retrospectively reviewed data for 73 consecutive patients with unresectable distal MBO who underwent endoscopic SEMS placement for the first time at our institution between April 2014 and March 2016. We compared time to RBO of SEMS placement above the papilla (intraductal placement) with SEMS placement across the papilla (transpapillary placement). In the intraductal placement group, we also compared time to RBO of placement without EST with placement with EST.

Results Endoscopic SEMS placement was performed in 30 patients with intraductal placement and in 43 patients with transpapillary placement. The median time to RBO was significantly longer with intraductal placement (307 days) than with transpapillary placement (161 days) $(P=0.022)$. Complication rates did not differ between the two groups. In both univariate and multivariate analysis, intraductal placement was an independent factor contributing to prolonged time to RBO. In intraductal placement, time to RBO was significantly longer in SEMS placement without EST than with EST (363 days vs. 227 days, respectively; $P=$ 0.026).

Conclusions Intraductal SEMS placement, especially without EST for distal MBO contributed to longer time to RBO.

\section{Introduction}

Endoscopic stent placement is an established palliative treatment for patients with distal malignant biliary obstruction (MBO), and self-expandable metallic stents (SEMS) have shown superiority over plastic stents in randomized trials [1,2]. Many comparative studies have evaluated covered vs. uncovered
SEMS [3-6]. In these studies, SEMS were placed across the papilla. Few studies have evaluated endoscopic SEMS placement above the papilla for extrahepatic MBO $[7,8]$. The merit of intraductal SEMS placement is believed to lie in preventing food impaction and bacterial contamination from the duodenum $[7,9]$. However, the usefulness of endoscopic SEMS placement above the papilla has not been fully examined. 
Some endoscopists often perform endoscopic sphincterotomy (EST) before SEMS placement to prevent pancreatitis following endoscopic retrograde cholangiopancreatography (ERCP). EST presumably decreases compression of the pancreatic duct orifice caused by transpapillary stent expansion [10]. However, in recent studies, no benefit of EST before transpapillary SEMS placement has been proven in patients with distal $\mathrm{MBO}$, especially with MBO due to pancreatic cancer [11-14]. EST is considered less necessary for intraductal SEMS placement because it does not cause compression of the pancreatic duct orifice. Moreover, EST prior to intraductal SEMS placement was reported to be associated with an increased incidence of acute cholangitis [15]. The safety and efficacy of intraductal SEMS placement without EST for distal MBO remains to be determined.

In this study, we compared intraductal SEMS placement with transpapillary SEMS placement in patients with unresectable distal MBO regarding the time to recurrent biliary obstruction (RBO). We also examined whether EST impacts the time to RBO following intraductal SEMS placement.

\section{Patients and methods}

\section{Patients}

We retrospectively reviewed data for 76 consecutive patients with unresectable distal MBO (defined as biliary obstruction located anywhere in the extrahepatic bile duct except for the hilar part) who underwent endoscopic SEMS placement for the first time between April 2014 and March 2016 at Osaka International Cancer Institute. Diagnoses of malignancy in all patients were cytopathologically proven before metallic stent placement. Distal MBO was confirmed in all patients according to laboratory data and imaging findings, including ERCP, abdominal enhanced computed tomography, and magnetic resonance cholangiopancreatography. We excluded three patients from this study because they had received duodenal stents $(n=1)$ or because they underwent concurrent placement of plastic stents and SEMS $(n=2)$. As a result, we analyzed data for 73 patients in this study. The present study was approved by the Institutional Review Board at Osaka International Cancer Institute (18050).

\section{Endoscopic procedures}

ERCP was performed under fluoroscopic guidance and using a side-viewing endoscope (JF260V, TJF260V; Olympus, Tokyo, Japan) under conscious sedation with midazolam and analgesic drugs (pentazocine or pethidine). All ERCP procedures were performed by endoscopists with more than 5 years' experience. Cannulation of the common bile duct was attempted by the wire-guided method with a conventional cannula (PR-104Q-1 cannula; Olympus Optical Co., Ltd., Tokyo, Japan or a conical tip cannula; MTW Endoskopie Manufaktur, Wesel, Germany). After biliary cannulation, cholangiography showed the location and the length of the biliary stricture. The guidewire was passed through the stricture and inserted into the common bile duct. EST was performed at the discretion of each physician. The main purposes of EST were to facilitate endoscopic reinterven- tion when RBO occurred and/or to decrease the risk of postERCP pancreatitis due to the lack of main pancreatic duct dilatation. Intraductal placement required a distal margin of more than $10 \mathrm{~mm}$ above the duodenal papilla, and the stent position was decided under fluoroscopic guidance. The types of SEMS (fully-covered/partially-covered/uncovered), stent manufacturer and the position of the SEMS (intraductal placement/ transpapillary placement) were decided at the discretion of the physician according to the length and position of the biliary stricture (cholangiographic findings).

\section{Definitions}

Enrollment was defined as the date of the initial SEMS placement, and we reviewed patients' clinical information, including fever and pain data, from their medical records. Blood peripheral examination and biochemistry was performed before and after ERCP in all patients. The primary outcome was the time to RBO, which was defined as a composite endpoint of stent occlusion or migration. We defined the time to RBO as the time from the initial SEMS placement to the recurrence of symptomatic biliary obstruction according to Tokyo criteria 2014 [16]. Other outcomes were technical success, clinical success, complications, risk factors associated with biliary obstruction, and overall survival time (the time from the initial SEMS placement to death). Technical success was defined as appropriate stent positioning for the stricture. Clinical success was defined as a decrease in bilirubin level to less than $75 \%$ of the pretreatment value when bilirubin level was $>3.0 \mathrm{mg} / \mathrm{dL}$ or maintenance of bilirubin level when the value was $<3.0 \mathrm{mg} / \mathrm{dL}$. Cholangitis was diagnosed based on Tokyo Guidelines 2018 [17]. Complications were defined as procedure-related events according to Tokyo criteria 2014 [16].

\section{Statistical analysis}

We compared baseline characteristics between the groups using the Mann-Whitney $U$ test for continuous variables and the Chi-square or Fisher's exact test for categorical variables. Statistical significance was set at $P<0.05$. Time to RBO and patient survival were analyzed using the Kaplan-Meier method, and differences were evaluated using the log-rank test.

Using the Cox proportional hazards model, univariate and multivariate analyses were performed to identify significant factors associated with longer time to RBO. The following nine variables were examined: age, sex, primary disease, duodenal invasion, treatment, presence of cholangitis at the time of SEMS placement, EST, the method of SEMS placement (intraductal or transpapillary), and the history of endoscopic biliary drainage before SEMS placement. Factors with a $P<0.30$ in the univariate analysis were entered into the multivariate Cox models Hazard ratios (HR) and 95\% confidence intervals (Cls) were calculated. Statistical analyses were performed using IBM SPSS statistics for Windows version 20.0 (IBM Corp., Armonk, New York, United States). 


\section{Results}

\section{Patient characteristics}

Thirty- five patients (48\%) were men, and the median age was 68 years (range, $46-87$ years). MBO was caused by pancreatic cancer (65 patients; $89 \%$ ) and biliary tract cancer ( 8 patients; $11 \%)$. Fourteen patients (19\%) suffered from duodenal invasion caused by cancer. After SEMS placement, 58 patients (79\%) underwent chemotherapy and 15 patients (21\%) received best supportive care without chemotherapy. Twenty-two patients (32\%) had already undergone or underwent EST before SEMS placement. Fifty- two patients $(71 \%)$ had undergone endoscopic biliary drainage before SEMS placement; 42 patients had received a transpapillary plastic stent, and 10 patients had undergone endoscopic nasal biliary drainage (ENBD) before SEMS placement, while 21 patients had never received biliary drainage. Regarding SEMS placement, intraductal placement and transpapillary placement were performed in 30 patients $(41 \%)$ and in 43 patients (59\%), respectively. Among the 30 patients who underwent intraductal SEMS placement, seven patients (23\%) underwent concurrent ENBD to prevent early cholangitis secondary to papillary edema. ENBD was discontinued 1 day later in four patients, 2 days later in one patient, 3 days later in one patient, and 8 days later in one patient. No prophylactic pancreatic stent was used in any patient included in the current study.
- Table 1 is a comparison of characteristics between patients in the intraductal placement and transpapillary placement groups. We found no statistical difference in age, sex, primary disease, treatment, duodenal invasion, presence of cholangitis at the time of SEMS placement, or history of endoscopic biliary drainage before SEMS placement, between the two groups ( $\triangleright$ Table $\mathbf{1}$ ). In contrast, stent type (uncovered or covered) significantly differed between the two groups $(P<$ $0.001)$. The uncovered SEMS was mainly used in the intraductal placement group (83\%; 25/30) to decrease risk of migration, while the covered SEMS was mainly used in the transpapillary placement group $(84 \% ; 36 / 43)$ due to its removability. Moreover, the length between the lower end of the MBO and the ampulla was significantly longer in the group undergoing intraductal placement (intraductal placement, median: $23 \mathrm{~mm}$ [range: 11-47 mm] vs. transpapillary placement, median: $5 \mathrm{~mm}$ [range: $0-32 \mathrm{~mm}$ ]) $(P<0.001)$. The number of patients who had previously undergone EST or who underwent EST during SEMS placement was not significantly different between the two groups. Among 22 EST cases, 15 (seven intraductal and nine transpapillary) were performed at our hospital and all of them were minor EST. In the other six cases of EST performed at other hospitals at the time of endoscopic biliary drainage before referral to our hospital, the detailed information about EST (major or minor EST) was not obtained.

- Table 1 Comparison between intraductal placement and transpapillary placement of self-expandable metallic stents (SEMS).

\begin{tabular}{|c|c|c|c|}
\hline Factor & $\begin{array}{l}\text { Intraductal } \\
n=30\end{array}$ & $\begin{array}{l}\text { Transpapillary } \\
n=43\end{array}$ & $P$ value \\
\hline $\begin{array}{l}\text { The length between the lower end of the MBO and the ampulla (mm) } \\
\text { [median (range)] }\end{array}$ & $23(11-47)$ & $5(0-32)$ & $<0.001$ \\
\hline Stent type (uncovered/covered) & $25 / 5$ & $7 / 36$ & $<0.001$ \\
\hline Age (years) [median (range)] & $66(57-84)$ & $72(46-87)$ & 0.318 \\
\hline Sex (female/male) & $14 / 16$ & $24 / 19$ & 0.441 \\
\hline Primary disease (PC/BTC) & $25 / 5$ & $40 / 3$ & 0.178 \\
\hline Duodenal invasion (\%) & $17(5 / 30)$ & $21(9 / 43)$ & 0.649 \\
\hline Treatment (Chemotherapy/BSC) & $24 / 6$ & $34 / 9$ & 0.923 \\
\hline Presence of cholangitis at the time of SEMS placement (\%) & $60(18 / 30)$ & $69\left(29 / 42^{1}\right)$ & 0.427 \\
\hline EST (yes/no) & $10 / 20$ & $12 / 31$ & 0.619 \\
\hline History of endoscopic biliary drainage before SEMS placement (yes/no) & $20 / 10$ & $32 / 11$ & 0.472 \\
\hline Technical success rate $(\%)$ & $100(30 / 30)$ & $100(43 / 43)$ & \\
\hline Clinical success rate (\%) & $100(30 / 30)$ & $98(42 / 43)$ & 0.589 \\
\hline Complication rate $(\%)$ & $7(2 / 30)$ & $7(3 / 43)$ & 0.668 \\
\hline Rate of RBO (\%) & $43(13 / 30)$ & $52(23 / 43)$ & 0.393 \\
\hline \multicolumn{4}{|c|}{$\begin{array}{l}\text { PC, pancreatic cancer; BTC, biliary tract cancer; MBO, malignant biliary obstruction; BSC, best supportive care; EST, endoscopic sphincterotomy; PS: plastic stent; } \\
\text { ENBD, endoscopic nasobiliary drainage; RBO, recurrent biliary obstruction. } \\
\text { Statistically significant at } P<0.05 \text {. } \\
{ }^{1} \text { Missing data for one patient. }\end{array}$} \\
\hline
\end{tabular}


- Table2 Detailed information on self-expandable metallic stents used in the two groups.

\begin{tabular}{|c|c|c|}
\hline & Stent type & No. \\
\hline \multirow{5}{*}{$\begin{array}{l}\text { Intraductal } \\
\mathrm{n}=30\end{array}$} & BONASTENT (Standard Sci Tech) uncovered & 1 \\
\hline & EGIS (S\&G Biotech) uncovered & 2 \\
\hline & Niti-S ComVi (Taewoong) partially-covered & 5 \\
\hline & Niti-S uncovered stent (Taewoong) uncovered & 19 \\
\hline & Niti-S Large cell D-type stent (Taewoong) uncovered & 3 \\
\hline \multirow{8}{*}{$\begin{array}{l}\text { Transpapillary } \\
\mathrm{n}=43\end{array}$} & BONASTENT (Standard Sci Tech) fully-covered & 2 \\
\hline & EGIS (S\&G Biotech) fully-covered & 1 \\
\hline & EGIS (S\&G Biotech) uncovered & 4 \\
\hline & Niti-S ComVi (Taewoong) fully-covered & 10 \\
\hline & Niti-S ComVi (Taewoong) partially-covered & 1 \\
\hline & Niti-S uncovered stent (Taewoong) uncovered & 3 \\
\hline & SUPREMO (Taewoong) fully-covered & 19 \\
\hline & X-Suit NIR (Olympus) fully-covered & 3 \\
\hline
\end{tabular}

\section{Stent placement and complications}

In patients undergoing intraductal placement, an uncovered SEMS was placed in 25 patients, and a partially-covered SEMS was placed in five patients ( $\triangleright$ Table 1 ). In contrast, in patients undergoing transpapillary placement, 36 patients received covered SEMS (fully-covered: 35; partially-covered: 1), and seven patients received uncovered SEMS. Detailed information of the SEMS used in the two groups is shown in > Table 2. Technical success was achieved in all cases, and the clinical success rate was $99 \%$ (72/73) ( Table 1). For both technical and clinical success rates, there was no statistical difference between the two groups.

Five patients (7\%) suffered from complications caused by SEMS placement. In the intraductal placement group, two complications occurred, namely, one patient with cholangitis and one with liver abscess. In the transpapillary placement group, three complications occurred, namely, two patients with cholecystitis and one with liver hematoma. The complication rate was not different between the two groups (intraductal group: $7 \%$, 2/30; transpapillary group: 7\%, 3/43) (\Table 2). No procedure-related deaths occurred.

\section{RBO}

RBO developed in 36/73 patients and the rate was not significantly different between the two groups ( $\downarrow$ Table 2 ). In 30 patients in the intraductal placement group, RBO occurred in 13 patients (43\%), and 10 of 13 suffered RBO due to tumor ingrowth (77\%). Other reasons for RBO were hemobilia $(n=1)$, sludge without stone $(n=1)$, and sludge secondary to biliary duodenal leakage $(n=1)$. Among the 43 patients in the transpapillary placement group, RBO occurred in 23 patients (52\%); 13 of 23 patients developed RBO due to sludge without stone (57\%), and $7 / 23$ developed RBO caused by stent migration
$(30 \%)$. Other reasons were hemobilia $(n=2)$ and tumor ingrowth $(n=1)$. Stent migration occurred significantly more frequently in the transpapillary placement group (intraductal: $0 \%$ $(0 / 30)$ vs. transpapillary: $16 \%(7 / 43)(P=0.037)$. In contrast, tumor ingrowth occurred significantly more frequently in the intraductal placement group (intraductal: $33 \%$ (10/30) vs. transpapillary: $2 \%(1 / 43)(P<0.001)$.

Regarding time to RBO, the median time to RBO in the intraductal placement group was significantly longer than that in the transpapillary placement group (307 days vs. 161 days, respectively; $P=0.022$ ) ( $\triangleright$ Fig. 1 ). We evaluated the probability of RBO as potential prediction using Cox proportional-hazards analyses. In the univariate analysis of nine variables, intraductal placement was significantly associated with longer time to RBO (HR: 0.446, 95 \% Cl: 0.219-0.908, $P=0.026$ ) ( Table 3). In multivariate analysis using the three variables with $P<0.3$ in the univariate analysis (primary disease, EST, and stent placement), intraductal placement was identified as a statistically significant independent factor for longer time to RBO (HR: 0.469, $95 \%$ Cl: 0.229-0.959, $P=0.038$ ) ( $\triangleright$ Table 3). Regarding overall survival, median survival time was not significantly different between the two groups (intraductal: 296 days vs. transpapillary: 302 days, $P=0.891)$. In the cases where the distal margin was $\geq 11 \mathrm{~mm}$ above the papilla, the median time to RBO in the intraductal placement group was significantly longer than that in the transpapillary placement group (intraductal: 307 days vs. transpapillary: 135 days; $p=0.005$ ) (Supplementary Fig. 1).

Finally, we examined the impact of EST on time to RBO in the intraductal placement group because intraductal placement without EST could effectively reduce the risk of duodenobiliary reflux. In the intraductal placement group, the median time to RBO for patients without EST was significantly longer than that for patients with EST (no-EST: 363 days vs. EST: 227 days; $P=$ 0.026 ) ( $\triangleright$ Fig. 2). EST was the only factor identified as being sig- 


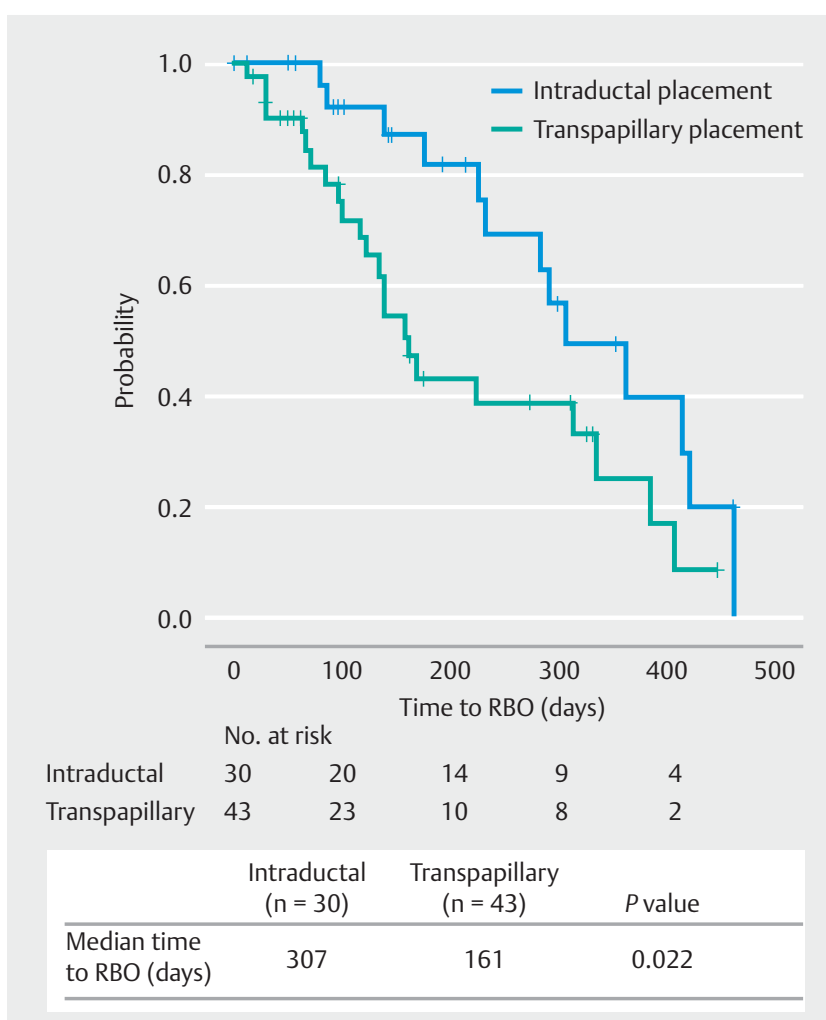

- Fig. 1 Comparison of the time to RBO between intraductal placement and transpapillary placement. RBO, recurrent biliary obstruction.

nificantly associated with time to RBO (HR: 3.488, $95 \% \mathrm{Cl}$ : 1.090-11.157; $P=0.035$ ) (Supplementary Table 1). The 20 patients without EST experienced a total of seven RBO events (tumor ingrowth $(n=6)$, sludge secondary to biliary duodenal leakage $(n=1))$ and one complication (cholangitis). The 10 patients with EST experienced a total of six RBO events (tumor ingrowth $(n=4)$, hemobilia $(n=1)$, sludge without stone $(n=1)$ ) and one complication (liver abscess).

In contrast, the median time to RBO was not significantly different for patients without EST compared with patients with EST in the transpapillary placement group (no-EST: 161 days vs. EST: 130 days; $P=0.751)$. These results suggest that intraductal placement without EST may be a more efficient method to prolong time to RBO.

\section{Discussion}

Endoscopic SEMS placement is an established standard treatment for palliative drainage of distal MBO [18]. SEMS are usually placed across the duodenal papilla in patients with unresectable distal MBO, but the optimal choice of SEMS has not been established. In a meta-analysis of endoscopic transpapillary SEMS placement for distal MBO, no significant difference was found between covered and uncovered SEMS regarding stent patency or between fully-covered vs. partially-covered SEMS
$[19,20]$. Therefore, a new strategy to extend the time to RBO is required for patients with distal MBO.

In this retrospective study, endoscopic intraductal placement of SEMS was significantly associated with longer time to RBO compared with transpapillary placement in patients with distal MBO, and multivariate analysis identified intraductal placement as an independent factor contributing to longer time to RBO ( $>$ Fig. 1 and $>$ Table 3 ). Intraductal SEMS placement is expected to prevent duodenobiliary reflux $[7,21]$, which is a common phenomenon after transpapillary SEMS placement and is considered a predisposing factor for stent occlusion and cholangitis [9, 22]. However, few reports have evaluated endoscopic intraductal placement of SEMS for MBO. One prospective study of fully-covered SEMS for extrahepatic MBO revealed favorable results following endoscopic intraductal placement (stent patency: 297 days) [7]. In endoscopic SEMS placement for unresectable hilar cholangiocarcinoma, intraductal placement showed a significant advantage for time to RBO over transpapillary placement [23]. To our knowledge, the current study is the first report to demonstrate the superiority of intraductal placement of SEMS for distal MBO compared with transpapillary placement.

Intraductal placement requires distance from the lower end of the MBO to the duodenal ampulla. Previous studies set this distance at a minimum of 5 to $15 \mathrm{~mm}[7,8]$. In the current study, the minimum distal margin was $11 \mathrm{~mm}$ above the papilla, in the intraductal placement group. The technical difficulty of intraductal placement may be a concern, but technical success was accomplished in all of our patients. Furthermore, there was no significant difference in complication rates between intraductal placement and transpapillary placement. These results suggested that intraductal placement can be accomplished safely in cases where the distal margin is $\geq 11 \mathrm{~mm}$ above the papilla.

Clinical success was also achieved in all patients undergoing intraductal placement. Endoscopic procedures may cause duodenal papillary edema and bile flow obstruction. Therefore, in approximately $20 \%$ of patients in our intraductal placement group, we used concurrent ENBD to prevent early cholangitis secondary to duodenal papillary edema; ENBD was discontinued a few days after placement. As a result, no early cholangitis was observed in the intraductal placement group. Simultaneous ENBD placement may not be essential, but the procedure can be a useful option to decrease the risk of early cholangitis.

Even though stent type and length between the lower end of the $\mathrm{MBO}$ and the ampulla might be important factors affecting the time to RBO, they were significantly different between the intraductal placement group and transpapillary placement group $(P<0.001)$ ( $>$ Table 1$)$. We believe that these two factors are not applicable for multivariate analysis because the inclusion of these procedure-related factors resulted in over-adjustment of bias. In the intraductal placement group, uncovered SEMS were mainly used to reduce the risk of stent migration [19]. In contrast, covered SEMS were mainly used in the transpapillary placement group due to its removability. Stent migration occurred more frequently in the transpapillary placement group than the intraductal placement group, while stent in- 
- Table 3 Univariate and multivariate analysis of factors associated with time to RBO.

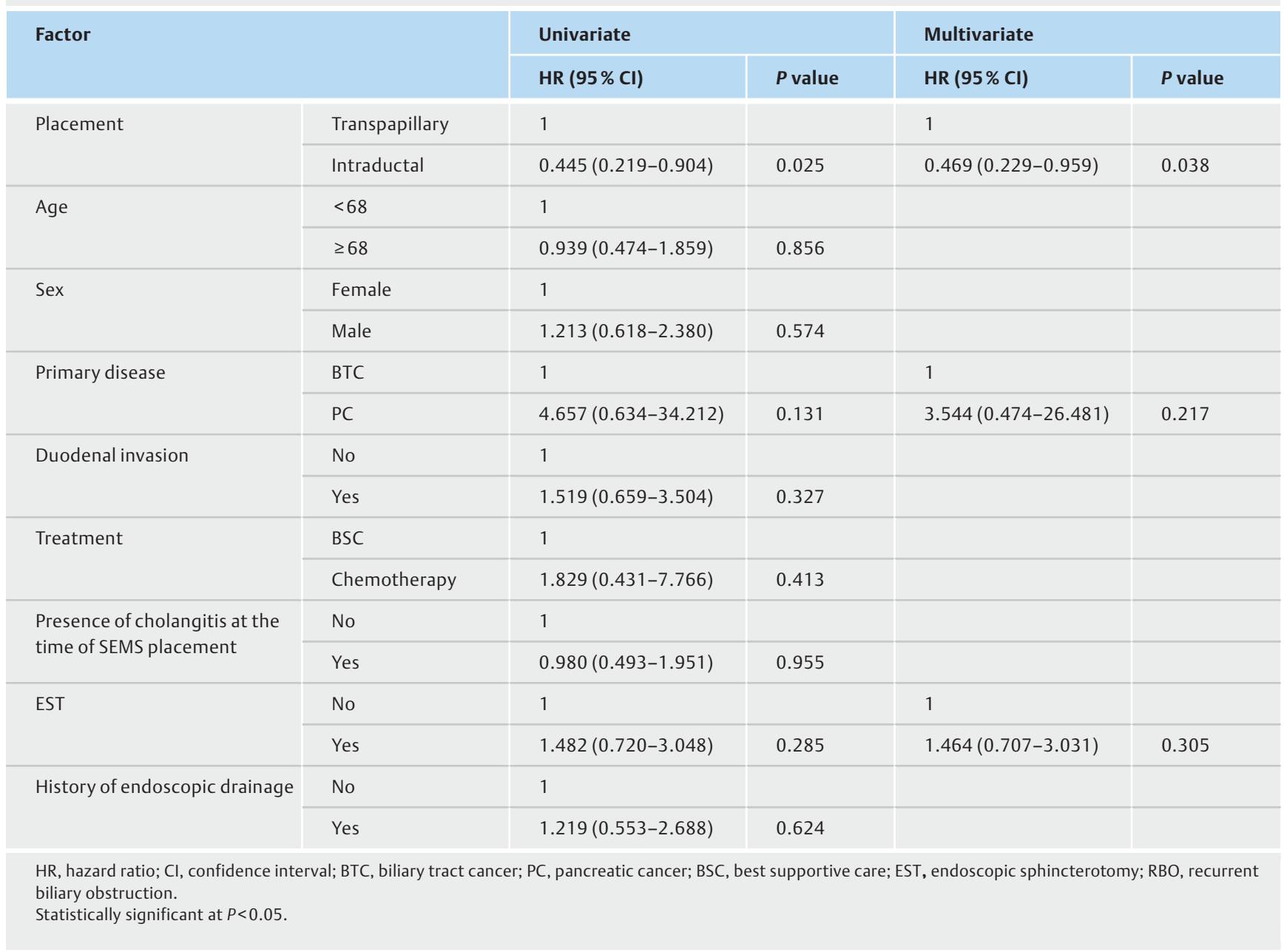

growth was more common in the intraductal placement group than the transpapillary placement group. These results suggest that the differences in stent types between the two groups were not associated with the time to RBO, as the merit of preventing tumor ingrowth is offset by the stent migration, with covered SEMS. A previous meta-analysis including a larger number of patients with distal MBO found no significant differences between patients treated with covered SEMS and those treated with uncovered SEMS [19]. Taken together, longer time to RBO in the intraductal placement group was likely to be due to the position of stent placement rather than the intergroup differences in stent type.

The significant difference between the transpapillary placement group and the intraductal placement group in length between the lower end of the MBO and the ampulla was unavoidable, as intraductal SEMS placement was not performed when the distance was $<11 \mathrm{~mm}$. Therefore, the comparison of the time to RBO between the intraductal placement group and the transpapillary placement group was limited to the cases where the distal margin was $\geq 11 \mathrm{~mm}$ above the papilla (Supplementary Fig. 1). The intraductal placement group exhibited a significantly longer time to RBO than the transpapillary placement group. This result supports the merit of intraductal placement compared with transpapillary placement, irrespective of the length between the lower end of the MBO and the ampulla.

It is noteworthy that intraductal placement without EST was associated with significantly longer time to RBO compared with EST ( Fig.2). EST was the only identified factor significantly associated with time to RBO in the intraductal placement group (Supplementary Table 1). EST is performed to decrease risk of pancreatitis, but the merit of EST prior to SEMS placement has not been proven [11-14].

Furthermore, damage to the sphincter of Oddi caused by EST could cause duodenobiliary reflux and stent occlusion [24, 25]. Interestingly, time to RBO differed significantly between EST and no-EST in the intraductal placement group, but not in the transpapillary placement group ( $\mathbf{F i g . 2}$ ). These results suggested that preserving function in the sphincter of Oddi profoundly influences time to RBO by preventing duodenobiliary reflux. Intraductal SEMS placement without EST could be the best way to prolong time to RBO if the length from the lower end of the MBO to the ampulla is not too short.

This study had limitations, namely, the single-center, nonrandomized, retrospective design. The method of SEMS placement (intraductal or transpapillary), stent type (covered or uncovered), stent brand, and decision to perform EST was per- 


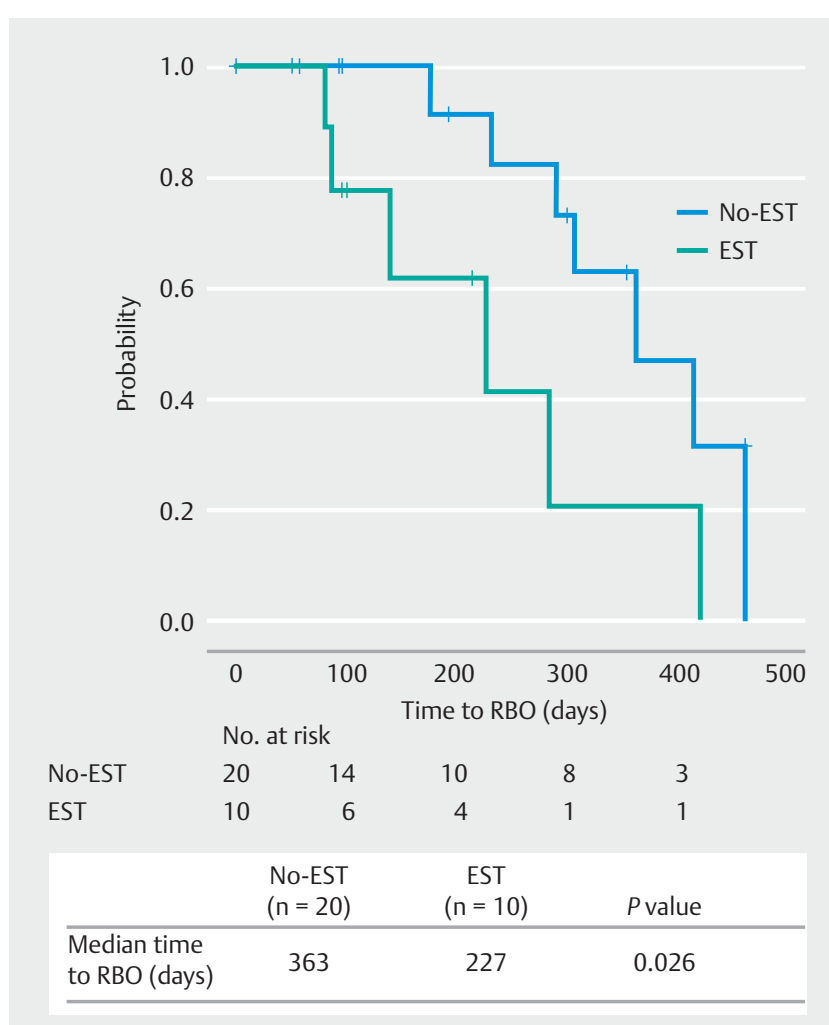

- Fig. 2 Comparison of the time to RBO between no-EST and EST in the intraductal placement group. $\mathrm{RBO}$, recurrent biliary obstruction; EST, endoscopic sphincterotomy.

formed based on the judgement of each physician in accordance with the length and position of the biliary stricture. The SEMS used in the current study were supplied by several manufacturers ( $\triangleright$ Table 2 ), and had different characteristics including axial forces and radial forces. Although the method of SEMS placement (intraductal vs. transpapillary) remained an independent prognostic factor for the time to RBO after adjustment using multivariate analysis, a prospective study with predetermined protocols is required to confirm the superiority of intraductal placement over transpapillary placement.

\section{Conclusion}

In conclusion, time to RBO was significantly longer with intraductal SEMS placement for distal MBO, and intraductal SEMS placement was identified as an independent factor associated with longer time to RBO. Intraductal SEMS placement without EST could be the optimal method to extend time to RBO because this method does not disrupt the sphincter mechanism and does not cause duodenobiliary reflux. Further prospective, large-scale studies are required to evaluate the merit of intraductal SEMS placement without EST.
The authors declare that they have no conflict of interest.

\section{References}

[1] Davids PH, Groen AK, Rauws EA et al. Randomised trial of self-expanding metal stents versus polyethylene stents for distal malignant biliary obstruction. Lancet 1992; 340: 1488-1492

[2] Wagner H], Knyrim K, Vakil N et al. Plastic endoprostheses versus metal stents in the palliative treatment of malignant hilar biliary obstruction. A prospective and randomized trial. Endoscopy 1993; 25: 213-218

[3] Isayama $H$, Komatsu $Y$, Tsujino $T$ et al. A prospective randomised study of "covered" versus "uncovered" diamond stents for the management of distal malignant biliary obstruction. Gut 2004; 53: 729734

[4] Kitano M, Yamashita Y, Tanaka K et al. Covered self-expandable metal stents with an anti-migration system improve patency duration without increased complications compared with uncovered stents for distal biliary obstruction caused by pancreatic carcinoma: a randomized multicenter trial. Am J Gastroenterol 2013; 108: 1713-1722

[5] Li ], Li T, Sun P et al. Covered versus uncovered self-expandable metal stents for managing malignant distal biliary obstruction: a meta-analysis. PLoS One 2016; 11: e0149066

[6] Conio M, Mangiavillano B, Caruso A et al. Covered versus uncovered self-expandable metal stent for palliation of primary malignant extrahepatic biliary strictures: a randomized multicenter study. Gastrointest Endosc 2018; 88: 283-291

[7] Choi H, Moon J, Lee Y et al. Evaluation of a newly modified nonflared fully covered metal stent, $12 \mathrm{~mm}$ in diameter, for intraductal placement in patients with malignant biliary strictures: a feasibility study. Endoscopy 2016; 48: 625-631

[8] Cho JN, Han J, Kim HG et al. Prospective randomized trial comparing covered metal stent placed above and across the sphincter of oddi in malignant biliary obstruction. Gastrointest Endosc 2013; 77: AB139AB140

[9] Okamoto T, Fujioka S, Yanagisawa S et al. Placement of a metallic stent across the main duodenal papilla may predispose to cholangitis. Gastrointest Endosc 2006; 63: 792-796

[10] Okano N, Igarashi Y, Kishimoto Y et al. Necessity for endoscopic sphincterotomy for biliary stenting in cases of malignant biliary obstruction. Dig Endosc 2013; 25: 122-125

[11] Hayashi T, Kawakami H, Osanai M et al. No benefit of endoscopic sphincterotomy before biliary placement of self-expandable metal stents for unresectable pancreatic cancer. Clin Gastroenterol Hepatol 2015; 13: 1151-1158

[12] Banerjee N, Hilden K, Baron TH et al. Endoscopic biliary sphincterotomy is not required for transpapillary sems placement for biliary obstruction. Dig Dis Sci 2011; 56: 591-595

[13] Kawakubo K, Isayama H, Nakai Y et al. Risk factors for pancreatitis following transpapillary self-expandable metal stent placement. Surg Endosc 2012; 26: 771-776

[14] Mangiavillano B, Montale A, Frazzoni L et al. Endoscopic biliary selfexpandable metallic stent in malignant biliary obstruction with or without sphincterotomy: systematic review and meta-analysis. Endosc Int Open 2019; 7: E26-E35

[15] Zhou H, Li L, Zhu F et al. Endoscopic sphincterotomy associated cholangitis in patients receiving proximal biliary self-expanding metal stents. Hepatobiliary Pancreat Dis Int 2012; 11: 643-649

[16] Isayama H, Hamada T, Yasuda I et al. Tokyo criteria 2014 for transpapillary biliary stenting. Dig Endosc 2015; 27: 259-264 
[17] Yokoe M, Hata J, Takada T et al. Tokyo Guidelines 2018: diagnostic criteria and severity grading of acute cholecystitis (with videos).

J Hepatobiliary Pancreat Sci 2018; 25: 41-54

[18] Dumonceau J-M, Tringali A, Papanikolaou I et al. Endoscopic biliary stenting: indications, choice of stents, and results: European Society of Gastrointestinal Endoscopy (ESGE) Clinical Guideline - Updated October 2017. Endoscopy 2018; 50: 910-930

[19] Tringali A, Hassan C, Rota M et al. Covered vs. uncovered self-expandable metal stents for malignant distal biliary strictures: a systematic review and meta-analysis. Endoscopy 2018; 50: 631-641

[20] Kogure H, Ryozawa S, Maetani I et al. A prospective multicenter study of a fully covered metal stent in patients with distal malignant biliary obstruction: WATCH-2 Study. Dig Dis Sci 2018; 63: 2466-2473

[21] Huang $X$, Shen L, Jin Y et al. Comparison of uncovered stent placement across versus above the main duodenal papilla for malignant biliary obstruction. J Vasc Interv Radiol 2015; 26: 432-437
[22] Misra SP, Dwivedi M. Reflux of duodenal contents and cholangitis in patients undergoing self-expanding metal stent placement. Gastrointest Endosc 2009; 70: 317-321

[23] Kubota K, Hasegawa S, Iwasaki A et al. Stent placement above the sphincter of Oddi permits implementation of neoadjuvant chemotherapy in patients with initially unresectable Klatskin tumor. Endosc Int Open 2016; 04: E427-E433

[24] Zhang R, Luo H, Pan Y et al. Rate of duodenal-biliary reflux increases in patients with recurrent common bile duct stones: evidence from barium meal examination. Gastrointest Endosc 2015; 82: 660-665

[25] Kurita A, Kodama Y, Minami R et al. Endoscopic stent placement above the intact sphincter of Oddi for biliary strictures after living donor liver transplantation. J Gastroenterol 2013; 48: 1097-1104 